Commun. Korean Math. Soc. 20 (2005), No. 2, pp. 231-238

\title{
THE MASS FORMULA OF ORDERS OVER A DYADIC LOCAL FIELD
}

\author{
Sungtae Jun and Insuk KIM
}

\begin{abstract}
In this paper, we study the arithmetic properties of orders in a quaternion algebra over a dyadic local field and we find the mass formula of orders.
\end{abstract}

\section{Introduction}

A primitive order in a quaternion algebra over a number field $F$ is an order which contains the ring of integers in a quadratic extension field of $F$. Locally, there are two types of quaternion algebras over a local field $k$, i.e., a division algebra and a $2 \times 2$ matrix algebra. In these quaternion algebras over a local field, primitive orders can be classified into three types. Namely, an order in a quaternion division algebra which contains the ring of integers of a quadratic extension field of $k$ is called primitive. In a $2 \times 2$ matrix algebra, there are two types of primitive orders. One is an order which contains $\mathcal{O} \times \mathcal{O}$ where $\mathcal{O}$ is the ring of integers in $k$ and the other is an order which contains the ring of integers of a quadratic extension field of $k$.

Primitive orders in $2 \times 2$ matrix algebra which contain $\mathcal{O} \times \mathcal{O}$ where $\mathcal{O}$ is the ring of integers were studied by Hijikata [4]. Primitive orders in a division algebra, so called "special orders", were studied by Hijikata, Pizer and Shemanske [5]. The remaining type of primitive orders was studied by Brezinski only on a nondyadic local field [2].

In this paper, we will study the arithmetic properties of third type of orders and compute the Mass formula of the primitive orders in a $2 \times 2$ matrix algebra containing the ring of integers of a quadratic extension field of a dyadic local field $k$, which is the remaining type of primitive orders not studied by Brezinski.

Received April 05, 2004.

2000 Mathematics Subject Classification: 11R52.

Key words and phrases: order, mass formula, dyadic local field.

The second author is supported by Wonkwang University in 2005. 


\section{Preliminaries}

Throughout this paper, we assume that $k$ is a dyadic local field. In this section we summarize the arithmetic theory of dyadic local fields . Let $\mathcal{O}=\mathcal{O}_{k}$ denote the ring of integers in $k, P=P_{k}$ the maximal ideal of $\mathcal{O}$. By $\Delta(\alpha)$, we denote the discriminant of $\alpha$.

$$
\Delta(\alpha)=\operatorname{Tr}(\alpha)^{2}-4 N(\alpha),
$$

where $\operatorname{Tr}$ and $\mathrm{N}$ are the trace and norm of $L$ over $k$ where $L$ is a quadratic extension field of $k$. If $\Gamma$ is an $\mathcal{O}$ algebra of rank 2 contained in $L$, then $\Gamma=\mathcal{O}+\mathcal{O} x$ and the discriminant of $\Gamma$ is

$$
\Delta(\Gamma)=\Delta(x) \bmod U^{2},
$$

where $U$ is the set of all units in $\mathcal{O}$.

Let $\mathcal{O}^{2}-4 \mathcal{O}=\left\{s^{2}-4 n \mid s, n \in \mathcal{O}\right\}$. Then we consider the set of all possible discriminants, $\left(\mathcal{O}^{2}-4 \mathcal{O}\right) / U^{2}$.

Definition 1. Let $\Delta_{\sigma}=\left(\left(\mathcal{O}^{2}-4 \mathcal{O}\right) \cap \pi^{\sigma} U\right) / U^{2}$ for $\sigma=0,1,2, \cdots$,

$$
\begin{aligned}
& \Delta_{0}^{*}=\Delta_{0}-\{1\}, \Delta_{1}^{*}=\Delta_{1}, \\
& \Delta_{\sigma}^{*}=\Delta_{\sigma}-\pi^{2} \Delta_{\sigma-2} .
\end{aligned}
$$

Note that $\Delta_{\sigma}^{*} \neq \phi$ only if $\sigma=2 \rho, 0 \leq \rho \leq e$, or $\sigma=2 e+1$ where $e=\operatorname{ord}_{k}(2)$. Let

$$
\Delta^{*}=\cup_{\sigma=0}^{\infty} \Delta_{\sigma}^{*}=\left(\cup_{\rho=0}^{e} \Delta_{2 \rho}^{*}\right) \cup \Delta_{2 e+1}^{*} .
$$

$\Gamma$ is a maximal order of a quadratic extension field of $k$ if and only if $\Delta(\Gamma) \in \Delta^{*}$. If $e>0$ and $1 \leq \rho \leq e$,

$$
\Delta_{2 \rho}^{*}=\pi^{2 \rho}\left(U^{2}+\pi^{2 e-2 \rho+1} U\right) / U^{2} .
$$

There is a bijective correspondence between the elements of $\Delta^{*}$ and quadratic extension fields of $k$ given by $\Delta(\Gamma) \rightarrow \Gamma \otimes \mathcal{O}$ for $\Delta(\Gamma)$ an element of $\Delta^{*}$.

LEMMA 2.1. Let $U$ be the set of all units in $\mathcal{O}$ and $e>0$. Then $U=U^{2}+P \supset U^{2}+P^{2} \supset \cdots \supset U^{2}+P^{2 e+1}=U^{2}$ and

$$
\left(U^{2}+P^{\sigma}\right) /\left(U^{2}+P^{\sigma+1}\right) \simeq \begin{cases}1 & \text { if } \sigma \text { is even and }<2 e \\ \mathbb{Z} / 2 \mathbb{Z} & \text { if } \sigma=2 e \\ \bar{k} & \text { if } \sigma \text { is odd }\end{cases}
$$

where $\bar{k}=\mathcal{O} / P$.

Proof. See Proposition 1.4 in [5]. 
Thus we can classify all quadratic extension fields of a dyadic local field $k$ as follows: $\Delta_{0}^{*}$ contains one point which corresponds to a unique unramified quadratic extension of $k$ and

$$
\Delta_{2 e+1}^{*}=\pi^{2 e+1} U / U^{2}
$$

contains $2 q^{2}$ points with $q=|\mathcal{O} / P|$.

Let $L$ be a quadratic extension field of $k$ and $x \rightarrow \bar{x}$ denote the conjugation of $L / k$. Further, let $\mathcal{O}_{L}$ be the ring of integers of $L, \mathcal{O}_{L}=$ $\mathcal{O}+\mathcal{O} \alpha$ for some $\alpha \in L$. Then $\Delta(L)=\Delta\left(\mathcal{O}_{L}\right)=\Delta(\alpha) U^{2}$, while $\Delta(\alpha)=$ $\operatorname{Tr}(\alpha)^{2}-4 \mathrm{~N}(\alpha)=(\alpha-\bar{\alpha})^{2}$. Whence, $\operatorname{ord}_{k}(\alpha-\bar{\alpha})^{2}=\operatorname{ord}_{k}(\Delta(\alpha))=$ $\operatorname{ord}_{k}(\Delta(L))$.

Definition 2. Let $L$ be a quadratic extension of $k$.

$$
t=t(L)=\operatorname{ord}_{k}(\Delta(L))-1 .
$$

REMARK. Note that if $L$ is an unramified extension field of $k$, then $t=-1$. On the other hand, if $L$ is a ramified extension field of a field $k$, then $t \geq 0$. Furthermore, if $k$ is a dyadic local field, then $0<t \leq 2 e$ by 2.3 and 1.3 in [5].

LEMMA 2.2. Let $L$ be a quadratic extension of $k$. If $x \in \mathcal{O}_{L}$, then $\operatorname{ord}_{L}(\Delta(x)) \geq \operatorname{ord}_{k}(\Delta(L))=t+1$.

PROOF. If $x=a+b \alpha \in \mathcal{O}_{L}=\mathcal{O}+\alpha \mathcal{O}$ with $a, b \in \mathcal{O}$, then $\operatorname{ord}_{k}(\Delta(x))=\operatorname{ord}_{k}\left(b^{2} \Delta(\alpha)\right) \geq \operatorname{ord}_{k}(\Delta(L))=t+1$.

\section{Orders in quaternion algebra}

Let $A$ be a quaternion algebra which is split over a dyadic local field $k$ (i.e. $A$ is isomorphic to $2 \times 2$ matrix algebra over $k$ ). and let $L$ be a quadratic extension field of $k$ contained in $A$. Then there exists an element $\xi$ in $A^{\times}$such that $A=L+\xi L$ and $x \xi=\xi \bar{x}$ for all $x \in L$. To see this clearly, we can identify $A$ with $\left\{\left(\begin{array}{cc}\alpha & \bar{\beta} \\ \beta & \bar{\alpha}\end{array}\right) \mid \alpha, \beta \in L\right\}$ and $L$ with $\left\{\left(\begin{array}{cc}\alpha & 0 \\ 0 & \bar{\alpha}\end{array}\right) \mid \alpha \in L\right\}$, where - is the conjugation of $L$ over $k$. Then $\xi$ is identified with $\left(\begin{array}{ll}0 & 1 \\ 1 & 0\end{array}\right)$. Hence the norm and the trace of an element in $A$ are defined as the determinant and the trace of corresponding element in 
$\left\{\left(\begin{array}{cc}\alpha & \bar{\beta} \\ \beta & \bar{\alpha}\end{array}\right) \mid \alpha, \beta \in L\right\}$. Also, $N(\xi)=-1$ implies that $\bar{\xi}=-\xi$. Further, for an arbitrary $x \in L, \overline{\xi x}=\bar{x} \bar{\xi}=-\xi x$.

If $\alpha$ is integral of degree 2 over $\mathcal{O}$ satisfying $\alpha^{2}-s \alpha+n=0$. We denote the discriminant of $\alpha$ by $\Delta(\alpha)=s^{2}-4 n$. Let $\mathcal{O}_{L}$ be the ring of integers in $L, P_{L}$ the prime ideal of $\mathcal{O}_{L}$. Let $\pi_{L}$ be the prime element of $P_{L}$. An order of a quaternion algebra $A$ is a lattice in $A$ which is also a subring containing the identity.

Proposition 3.1. Let the notation be as above. Let $R$ be an order of $A$ and $L$ a quadratic extension field in $A$. Then $R$ contains $\mathcal{O}_{L}$ if and only if

$$
R= \begin{cases}\mathcal{O}_{L}+\xi P_{L}^{n} & \text { if } L \text { is an unramified extension field } \\ \mathcal{O}_{L}+(1+\xi) P_{L}^{n-t-1} & \text { if } L \text { is a ramified extension field, or } \\ \mathcal{O}_{L}+(1-\xi) P_{L}^{n-t-1} & \end{cases}
$$

for some nonnegative integer $n$ and $t=t(L)$.

Proof. Suppose that $R$ is an order of $A$ which contains $\mathcal{O}_{L}$. Then $R=\mathcal{O}_{L}+y \mathcal{O}_{L}$ for some $y \in A . y \in R \subset A=L+\xi L$. Let $y=\alpha+\xi \beta$ for some $\alpha, \beta \in L$ and $\beta \neq 0$. If $x \in \mathcal{O}_{L}$, then $x y=x(\alpha+\xi \beta)=(x-\bar{x}) \alpha+y \bar{x}$. So $(x-\bar{x}) \alpha=x y-y \bar{x} \in R$ for any $x \in \mathcal{O}_{L}$. Since ord ord $_{L}(x-\bar{x}) \geq t+1$ by Lemma $2.2, \alpha \in P_{L}^{-t-1}$.

If $\alpha \in \mathcal{O}_{L}$, then $\beta \in \mathcal{O}_{L}$. For $N(y)=N(\alpha)-N(\beta) \in \mathcal{O}$. Let $n=\operatorname{ord}_{L} \beta$. Then $R=\mathcal{O}_{L}+\xi \beta \mathcal{O}_{L}=\mathcal{O}_{L}+\xi P_{L}^{n}$.

If $\alpha \notin \mathcal{O}_{L}$, then $\alpha \in P_{L}^{-t-1}-\mathcal{O}_{L}$. This is the case that $L$ is ramified. Let $\alpha=\pi_{L}^{-s} u$ and $\beta=\pi_{L}^{-s} w$ for $1 \leq s \leq t+1$. From $N(y)=N(\alpha)-$ $N(\beta) \in \mathcal{O}$, it is easy to see $N(u / w) \equiv 1 \bmod P$. This implies that $u / w \equiv \pm 1 \bmod P_{L}$. Thus $R$ is of the form, $\mathcal{O}_{L}+(1+\xi) P_{L}^{n-t-1}$ or $\mathcal{O}_{L}+(1-\xi) P_{L}^{n-t-1}$. The other direction of the proof is trivial.

Corollary 3.2. Let the notations be as above and $e=\operatorname{ord}_{k}(2)$. If $L$ is a ramified extension field of $k$,

$$
\begin{cases}\mathcal{O}_{L}+(1+\xi) P_{L}^{-t}=\mathcal{O}_{L}+(1-\xi) P_{L}^{-t} & \text { if } t=2 e \\ \mathcal{O}_{L}+(1+\xi) P_{L}^{-t-1}=\mathcal{O}_{L}+(1-\xi) P_{L}^{-t-1} & \text { if } t<2 e\end{cases}
$$

Proof. Let $\alpha+(1-\xi) \pi_{L}^{n-t-1} \beta \in \mathcal{O}_{L}+(1-\xi) P_{L}^{n-t-1}$. Then $\alpha+(1-$

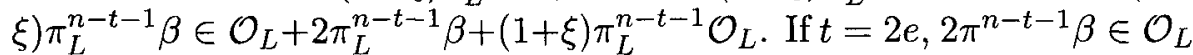
only if $n=0$. $\mathcal{O}_{L}+(1-\xi) P_{L}^{n-t-1}=\mathcal{O}_{L}+(1+\xi) P_{L}^{n-t-1}$ for $n \geq 1$. If $t<2 e, 2 \pi^{n-t-1} \beta \in \mathcal{O}_{L}$ for any nonnegative integer $n$. 
Let $\pi\left(\pi_{L}\right)$ be a prime element in the ring of integers in $k$ ( $L$, respectively). Then if $L$ is ramified, $\pi \equiv \pi_{L}^{2} \bmod \mathcal{O}_{L}^{\times}$and if $L$ is unramified, $\pi \equiv \pi_{L} \bmod \mathcal{O}_{L}^{\times}$. We now need new notations of orders for the next step.

Definition 3. Let $L$ be a quadratic extension field of $k$ and $\mathcal{O}_{L}$ its ring of integers. Then

(1) if $L$ is unramified, $R_{n}(L)=\mathcal{O}_{L}+\xi \pi_{L}^{n} \mathcal{O}_{L}$ for $n \geq 0$,

(2) if $L$ is ramified,

(a) if $t=2 e, R_{n}(L)=\mathcal{O}_{L}+(1+\xi) \pi_{L}^{n-t-1} \mathcal{O}_{L}$ for $n \geq 0$, or $\overline{R_{0}(L)}=\mathcal{O}_{L}+(1-\xi) \pi_{L}^{-t-1} \mathcal{O}_{L}$

(b) if $t<2 e, R_{n}(L)=\mathcal{O}_{L}+(1+\xi) \pi_{L}^{n-t-1} \mathcal{O}_{L}$ for $n \geq 0$.

REMARK. If $L$ is unramified, then the index $n$ of $R_{n}(L)$ is always an even number.

LEMMA 3.3. Let the notations be as above. Then

(1) if $L$ is unramified,

$$
\cdots \subset R_{2 n}(L) \subset R_{2 n-2}(L) \cdots \subset R_{0}(L),
$$

(2) if $L$ is ramified, $\cdots \subset R_{n}(L) \subset R_{n-1}(L) \cdots \subset R_{1}(L) \subset\left\{\begin{array}{l}\frac{R_{0}(L)}{R_{0}(L)} \text {. }\end{array}\right.$

Proof. This is immediate from Definition 3.

Proposition 3.4. Let $L$ be a ramified quadratic extension field of $k$ and $t=2 e$. Then $R_{n}(L) \approx\left(\begin{array}{cc}\mathcal{O} & \mathcal{O} \\ P^{n} & \mathcal{O}\end{array}\right)$ for $n=0,1$.

Proof. By the proof of Corollary 3.2, $R_{0}(L) \cap \overline{R_{0}(L)}=R_{1}(L)$. By Hijikata's results ([4], 2.2 p.65), $R_{1}(L) \approx\left(\begin{array}{cc}\mathcal{O} & \mathcal{O} \\ P^{n} & \mathcal{O}\end{array}\right)$ for some nonnegative integer $n$. Since $R_{1}(L)$ is the second largest order contained in the maximal order, $R_{0}(L) \approx\left(\begin{array}{ll}\mathcal{O} & \mathcal{O} \\ \mathcal{O} & \mathcal{O}\end{array}\right)$, we conclude that $n=1$. Clearly, $R_{1}(L) \approx\left(\begin{array}{ll}\mathcal{O} & \mathcal{O} \\ P & \mathcal{O}\end{array}\right)$

Theorem 3.5. Let the notations be as above and $p=|\mathcal{O} / P|$. If $L$ is unramified, then $\left|R_{2 k}^{\times} / R_{2 k+2}^{\times}\right|=p^{2}$ for $k \geq 1$ and $\left|R_{0}^{\times} / R_{2}^{\times}\right|=p^{2}-p$.

Proof $k>0, \alpha+\xi \beta \in R_{2 k}^{\times}$implies that $\alpha \in \mathcal{O}_{L}$ and $\beta \in P_{L}^{k}$. $N(\alpha+\xi \beta)=N(\alpha)-N(\beta) \in \mathcal{O}^{\times}$. That is, $\alpha \in \mathcal{O}_{L}^{\times}$. Now $R_{2 k}^{\times}=$ $\left(1+\xi P_{L}^{2 k}\right) R_{2 k+2}^{\times} .\left(1+\xi \beta_{1}\right) \overline{\left(1+\xi \beta_{2}\right)}=\left(1+\xi \beta_{1}\right)\left(1-\xi \beta_{2}\right)=1-\overline{\beta_{1}} \beta_{2}+$ 
$\xi\left(\beta_{1}-\beta_{2}\right) \in R_{2 k+2}^{\times}$. Thus $\left(1+\xi \beta_{1}\right) R_{2 k+2}^{\times}=\left(1+\xi \beta_{2}\right) R_{2 k+2}^{\times}$if and only if $\beta_{1} \equiv \beta_{2} \bmod P_{L}^{k+1} . R_{2 k}^{\times} / R_{2 k+2}^{\times} \approx P_{L}^{k} / P_{L}^{k+1}$ by the map $\phi(1+$ $\left.\xi \pi^{-t-1} \beta\right)=\beta$. Since $\left|P_{L}^{k} / P_{L}^{k+1}\right|=\left|\mathcal{O}_{L} / P_{L}\right|=p^{2},\left|R_{2 k}^{\times} / R_{2 k+2}^{\times}\right|=p^{2}$. Next, if $\alpha+\xi \beta \in R_{0}^{\times}$, then $N(\alpha)-N(\beta) \in \mathcal{O}^{\times}$. There are three cases to be classified.

(1) $\alpha \in \mathcal{O}_{L}^{\times}$and $\beta \in P_{L}$.

(2) $\beta \in \mathcal{O}_{L}^{\times}$and $\alpha \in P_{L}$.

(3) $\alpha \in \mathcal{O}_{L}^{\times}$and $\beta \in \mathcal{O}_{L}^{\times}$and $N(\alpha)-N(\beta) \notin P$.

Hence, $R_{0}^{\times}=R_{2}^{\times} \cup \xi R_{2}^{\times} \cup\left\{(1+\xi s) R_{2}^{\times} \mid s\right.$ a representative of $\mathcal{O}_{L} / P_{L}$ with $N(s) \not \equiv 1 \bmod P\}$. Let $\tilde{N}$ be a homomorphism from $\left(\mathcal{O}_{L} / P_{L}\right)^{\times} \rightarrow$ $(\mathcal{O} / P)^{\times}$induced by the norm of $\mathcal{O}_{L}$ to $\mathcal{O}$. Then $\left(\mathcal{O}_{L} / P_{L}\right)^{\times} / \operatorname{ker}(\tilde{N}) \simeq$ $(\mathcal{O} / P)^{\times}$. Hence $|\operatorname{ker}(\tilde{N})|=p+1$. We now conclude that $\left|R_{0}^{\times} / R_{2}^{\times}\right|=$ $1+1+\left(\left|\left(\mathcal{O}_{L} / P_{L}\right)^{\times}\right|-p-1\right)=p^{2}-p$.

THEOREM 3.6. If $L$ is ramified, then

(1) $\left|R_{0}^{\times} / R_{1}^{\times}\right|=p+1$.

(2) $\left|R_{n}^{\times} / R_{n+1}^{\times}\right|=p$ for $n \geq 1$.

Proof. Suppose that $n \geq 1$. Then $\alpha+(1+\xi) \beta \in R_{n}^{\times}$implies that $N(\alpha+\beta)-N(\beta) \in \mathcal{O}^{\times}$. That is, $N(\alpha)+\operatorname{Tr}(\alpha \bar{\beta}) \in \mathcal{O}^{\times}$, which implies $N(\alpha) \in \mathcal{O}^{\times}$and $\alpha \in \mathcal{O}_{L}^{\times}$. Now let $R_{n}^{\times}=\left(\mathcal{O}_{L}^{\times}+(1+\xi) \pi_{L}^{-t-1} P_{L}^{n}\right)=$ $\left(1+(1+\xi) \pi_{L}^{-t-1} P_{L}^{n}\right) R_{n+1}^{\times}$. Then we can define a map $\phi$ from $R_{n}^{\times} / R_{n+1}^{\times}$ to $P_{L}^{n} / P_{L}^{n+1}$ by $\phi\left(\{1+(1+\xi) \beta\} R_{n+1}^{\times}\right) \equiv \beta \bmod P_{L}^{n+1}$. Since $(1+(1+$ $\xi) \delta)^{-1}=(1+\operatorname{Tr}(\delta)-(1+\xi) \delta) / N(1+(1+\xi) \delta)$,

$$
\begin{aligned}
\{1+\operatorname{Tr}(\delta) & -(1+\xi) \delta\}\{1+(1+\xi) \beta\} \\
& =1+\operatorname{Tr}(\delta)+(1+\xi)\{-\delta-\operatorname{Tr}(\delta) \beta+(1+\operatorname{Tr}(\delta)) \beta \\
& =1+\operatorname{Tr}(\delta)-(1+\xi)(\beta-\delta) .
\end{aligned}
$$

Hence $\beta \equiv \delta \bmod P_{L}$ implies that $\phi\left(1+(1+\xi) \delta R_{n+1}^{\times}\right)=\phi(1+(1+$ $\left.\xi) \beta R_{n+1}^{\times}\right)$if and only if $(1+(1+\xi) \delta) R_{n+1}^{\times}=(1+(1+\xi) \beta) R_{n+1}^{\times} \cdot \phi$ is well defined. It is clear that $\tilde{\phi}$ is bijective. i.e. $R_{n}^{\times} / R_{n+1}^{\times} \approx P^{n} / P^{n+1}$. Hence (2) is proved.

By Proposition 3.4 and 2.2 in p.65 of [4] and direct computations, $\left|R_{0}^{\times} / R_{1}^{\times}\right|=\left(p^{2}-1\right) /(p-1)=p+1$.

Definition 4. Let $M$ be an order of a quaternion algebra $A$ over a number field $F$. Let $F_{p}=F \otimes \mathbb{Q}_{p}$ for a prime $p$ and $\mathcal{O}_{p}$ is the ring of integers in $F_{p} . M$ is said to be an order of level $q p^{n}$ if $M \otimes \mathcal{O}_{q}$ is the maximal order of $A \otimes F_{q}$ and for some integer $n \geq 0, M \otimes \mathcal{O}_{p}=R_{n}(L)$ is 
an order of $A \otimes F_{p} \simeq\left\{\left(\begin{array}{ll}a & b \\ c & d\end{array}\right) \mid a, b, c, d \in F_{p}\right\}$ which contains the ring of integers of a quadratic extension field of $F_{p}$.

Our final goal is to compute the mass formula of an order of level $p^{n} q$. First of all, we need a definition of the mass formula. Let $A$ be a quaternion algebra ramified at a prime $q$ and $\infty$. Let $M$ be an order of level $N$ for some integer $N$ and let $I_{1}, I_{2}, \cdots I_{H}$ be representatives of the left $M$-ideal classes.

Definition 5. Let the notations be as above. The right order $M_{i}$ of $I_{i}$ is defined by

$$
M_{i}=\left\{a \in A \mid I_{i} a \subset I_{i}\right\}
$$

Definition 6. The Mass formula for $M$ ideals where $M$ is an order of $A$ is given by

$$
\operatorname{Mass}(M)=2 \sum_{i=1}^{H} \frac{1}{\left|U\left(M_{i}\right)\right|}
$$

where $U\left(M_{i}\right)=M_{i}^{\times}$.

Finally we can compute the mass formula for orders, $R_{n}$.

THEOREM 3.7. Let $M$ be an order of level $p^{n} q$. Then

$$
\operatorname{Mass}(M)=\frac{1}{12}(q-1) \delta
$$

where $\delta= \begin{cases}\left(p^{2}-p\right) p^{n-2} & \text { if } L \text { is unramified } \\ (p+1) p^{n-1} & \text { if } L \text { is ramified }\end{cases}$

Proof. Let $M^{0}$ be a maximal order in $A$ containing $M$. Then as in Proposition 24 and Proposition 25 in [9] p.685,

$$
\operatorname{Mass}(M)=\operatorname{Mass}\left(M^{0}\right)\left(\left[U\left(M^{0}\right): U(M)\right]\right)
$$

By Eichler's result [3], $\operatorname{Mass}\left(M^{0}\right)=\frac{1}{12}(q-1)$. Therefore we need to find $\left[U\left(M_{0}\right): U(M)\right]=\prod_{p}\left[U\left(M_{p}^{0}\right): U\left(M_{p}\right)\right]$. Since $M_{p}^{0}$ is a maximal order, by Definition $3, M_{p}^{0}=R_{0}(L)$ and $M_{p}=R_{n}(L)$. Hence, if $L$ is unramified extension field of $k$, then

$$
\begin{aligned}
{\left[U\left(M_{p}^{0}\right): U\left(M_{p}\right)\right] } & =\left[R_{0}^{\times}: R_{2}^{\times}\right] \cdots\left[R_{n-2}^{\times}: R_{n}^{\times}\right] \\
& =\left(p^{2}-p\right) p^{n-2} .
\end{aligned}
$$


If $L$ is ramified extension field of $k$, then

$$
\begin{aligned}
{\left[U\left(M_{p}^{0}\right): U\left(M_{p}\right)\right] } & =\left[R_{0}^{\times}: R_{1}^{\times}\right] \cdots\left[R_{n-1}^{\times}: R_{n}^{\times}\right] \\
& =(p+1) p^{n-1} .
\end{aligned}
$$

\section{References}

[1] A. Atkin and J. Lehner, Hecke operators on $\Gamma_{0}(N)$, Math. Ann. 185 (1970), $134-160$.

[2] J. Brezinski, On automorphisms of Quaternion orders J. Reine Angew. Math. 43 (1992), 102-109.

[3] M. Eichler, The basis problem for modular forms and the traces of Hecke operators, Springer-Verlag, Lecture Notes in Math. 320 (1972), 75-151.

[4] H. Hijikata, Explicit formula of the traces of the Hecke operators for $\Gamma_{0}(N), \mathrm{J}$. Math. Soc. Japan 26 (1974), 56-82,

[5] H. Hijikata, A. Pizer, and T. Shemanske, Orders in Quaternion Algebras, J. Reine Angew. Math. 394 (1989), 59-106.

[6] - The basis problem for modular forms on $\Gamma_{0}(N)$, Mem. Amer. Math. Soc. 82 (1982).

[7] T. Lam, The algebraic theory of quadratic forms, W.A. Benjamin, 1980

[8] A. Pizer, An Algorithm for computing modular forms on $\Gamma_{0}(N)$, J. Algebra 6 (1980), 340-390.

[9] - On the arithmetic of Quaternion algebras II, J. Math. Soc. Japan 28 (1976), 676-698.

[10] $\Gamma_{0}(N)$, Math. Ann. 226 (1977), 99-116.

[11] I. Reiner, Maximal orders, Academic Press, 1975.

[12] A. Weil, Basic number theory, Berlin, Hedelberg, New York: Springer, 1967.

Sungtae Jun

Division of Mathematics and Computer sicience

Konkuk University

Choongju 380-151, Korea

E-mail: sjun@kku.ac.kr

Insuk Kim

Division of Mathematics and Informational Statistics

Wonkwang University

Iksan 540-749, Korea

E-mail: iki@wonkwang.ac.kr 